\title{
ERRATUM
}

\section{ID FAMILY OF HELIX-LOOP-HELIX PROTEINS IN CANCER}

Jonathan Perk, Antonio Iavarone and Robert Benezra

Nature Rev. Cancer 5, 603-615 (2005)

On page 608 of this article, the statement 'latent membrane protein (LMP1, also known as TRAF3)' is incorrect. TRAF3 is the alternative name for LMP1-associated protein (LAP1) and is not an alternative name for LMP1. 